\title{
Electromechanical Modelling of High Power RF- MEMS Switches with Ohmic Contact
}

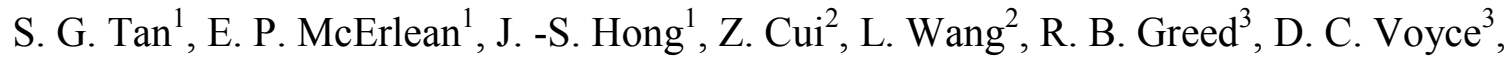 \\ ${ }^{1}$ Department of Electrical, Electronic and Computer Engineering, \\ Heriot-Watt University Edinburgh, EH14 4AS, U.K. \\ ${ }^{2}$ Central Microstructure Facility, Rutherford Appleton Laboratory, Didcot, U.K \\ ${ }^{3}$ Advanced Technology Research Centre, BAE SYSTEMS, Great Baddow, Chelmsford Essex CM2 8HN, U.K.
}

\begin{abstract}
This paper presents a study of the behaviour of electrically actuated RF-MEMS switches with ohmic contact. We will discuss about the relationship between the actuation voltage, displacement and the corresponding contact force experienced by the switch. We will demonstrate the linear behaviour of the switch when factors such as width or length of the switch arm are varied. Experimental results for DC actuation are also presented.
\end{abstract}

\section{INTRODUCTION}

Development in Micro-Electro-Mechanical Systems (MEMS) has promoted exciting advancements in microwave switching. This has led to the development of microdevices that involve contact during operation. MEMS technology holds much promise for military, space and commercial wireless communication systems [1]-[3]. This is due to their vast advantages as compared to their conventional semiconductor counterparts, such as very low insertion loss, very low power consumption and high linearity which result in lower signal distortion. However, most of reported RF MEMS switches are only capable of handling low microwave power, for instance, several hundred milliwatts, before device failure. Switch failure can be attributed to a number of mechanisms, such as stiction and buckling. It is envisaged that there is a great challenge in developing high power RF MEMS switches and both electromechanical and thermal modelling will play import role in such developments [4]-[6].

In this paper, we report recent development in high power RF MEMS switches and further work done in design and simulation of the mechanical properties of the RF MEMS switch, in particular, with regards to buckling. Experimental results for DC actuation are also presented. This work is part of the UK EPSRC research project (GR.S68910/01) on High power RF/microwave MEMS switches, involving three project partners: Heriot-Watt University, Rutherford Appleton Laboratories (RAL) and BAE Systems.

\section{CONFIGURATION}

In order to have high power RF MEMS capability, we have proposed a concept of high power RF MEMS switch incorporating a matrix or array of switching elements as shown in Fig. 1(a) [7]. By exploring power splitting /combining concept, an increase in rows of the switch matrix can effectively increase the power handling. While an increase in columns of the switch matrix will improve isolation. This approach allow us to configure the switch matrix to be tailored for different power and isolation requirements while keeping each individual switching element operating at low power flow and low actuation voltage. Some 2 by 2 or quadruplet switch configurations under investigation are illustrated in Fig. 1(b) to Fig. 1(d). All the switching elements have a metal ohmic contact.

In the case of Fig. 1(b) the switch simply consists of 4 individual switching elements without any mechanical connections. As for the quadruple switch design shown in Fig.1(c), 2 switching elements are mechanically linked by their 'heads' via silicon nitride material, making the mechanical structure (bridge type) more balanceable. In Fig 1(d), we can see that the quadruple switch is fully linked both on the heads (through silicon nitride) and also on the sides (via gold). Besides gaining the mechanical balance for all the four switching elements, this configuration allows applying DC voltage to all the top actuation electrodes through a single DC bias circuit.

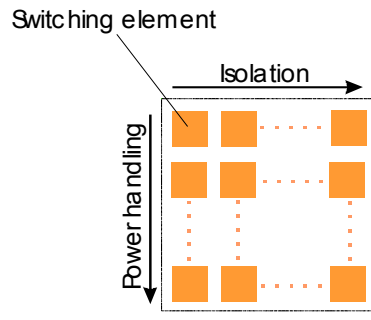

(a)

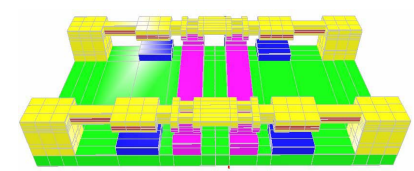

(c)

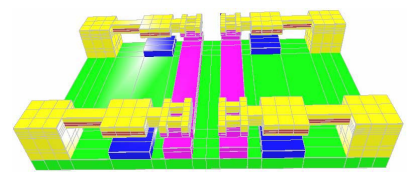

(b)

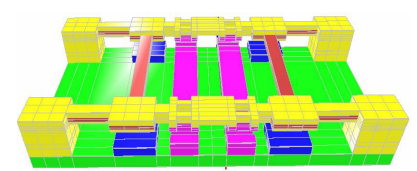

(d)
Fig. 1. High power RF MEMS switch configuration. (a) Concept. (b) Quadruple switch unlinked. (c) Quadruple switch partially linked. (d) Quadruple switch fully linked. 


\section{MODELLING}

Although there are some analytical formulations available for modelling RF MEMS switches [1], they result from very specific and simplified models and hence cannot provide an insight of mechanical behaviours. For developing high power RF MEMS switches, we have used a commercially available 3D modelling tool (IntelliSuite), which allows us to examine switch mechanical behaviours in more details, and to be able to study the buckling phenomenon. In this section, we will present the simulation results for developing the quadruplet switches of Fig. 1(b) and (c).

\section{A. Case 1}

Since the switch configuration of Fig. 1(b) is comprised of 4 independent switching elements, we can model each as a single RF MEMS switch as shown in Fig. 2(a), which has a typical size of $600 \mu \mathrm{m} \times 150 \mu \mathrm{m} \times$ $10 \mu \mathrm{m}$. This state corresponds to that when a zero actuation voltage is applied.
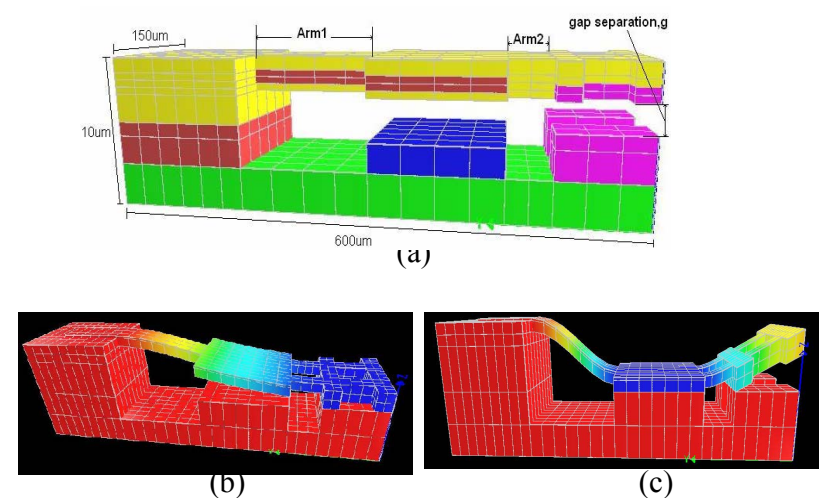

Fig. 2. (a) Unactuated RF MEMS single switch model. (b) Full contact state. (c) Buckling state.

It can be shown from the simulation that when the actuation voltage increases, the beam tip will make the first contact with the bottom RF transmission line, and then reach a full contact as shown in Fig. 2(b). It would be normally expected that further increasing the actuation voltage would increase the contact force, which is desired for high power handling. However, as the beam is not a rigid body, it has been found that subject to a larger actuation voltage the beam tip can starts to 'swing' out in the upwards direction and contact is broken. This failure occurrence is defined as the buckling as illustrated in Fig. 2(c).

The phenomena can also be observed from Fig. 3(a), where the vertical axis is the displacement of the beam tip. Two curves are plotted for the gap separation (initial beam height) $g=3$ and $4 \mu \mathrm{m}$ respectively. Each of the curves shows three distinct portions. The first portion shows the displacement increasing against the actuation voltage before the beam makes any contact with the base. The second portion is a straight line indicating that the beam tip is in contact with the RF transmission line on the substrate so that there is no change in the displacement. The third portion shows a decrease of the displacement, implying the beam tip has lost its RF contact. The actuation voltage at the transit point between the first and second portions is the minimum voltage required for making an $\mathrm{RF}$ contact, which increases against the initial height of the beam.

For high power handling, a good RF contact with full contact (largest contact area) and high contact force is highly desirable. The contact force for the this type of switching element is very complicated, and for the proposed model it can be estimated from the reaction force of the beam. Fig. 3 (b) depicts the reactions forces of the above two beams $(\mathrm{g}=3$ and $4 \mu \mathrm{m})$ vs. the actuation voltage. In general, the reaction forces increase against the actuation voltage. There is a clear transit point for each of the three curves shown, which corresponds to that when the beam makes the first contact. Before making any contact with the RF transmission line, the reaction force is only the spring or mechanical restoring force of the beam. After making contact, the contact force is generated and increased as the actuation voltage increases. However, the contact force is expected to be smaller than the reaction force since the later includes the restoring force of the beam. In any case, we can see from Fig. 3(b) that the contact force is in a range of a few to several-ten $\mathrm{uN}$.

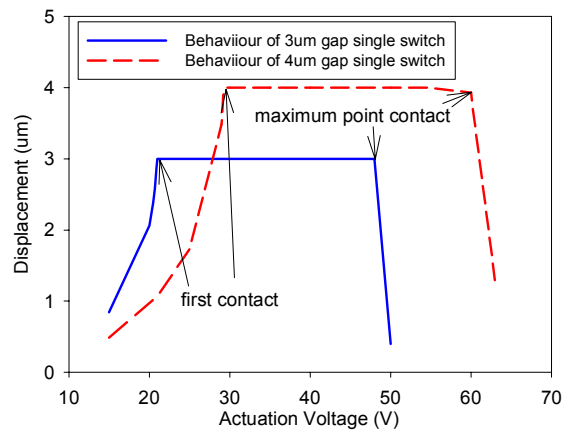

(a)

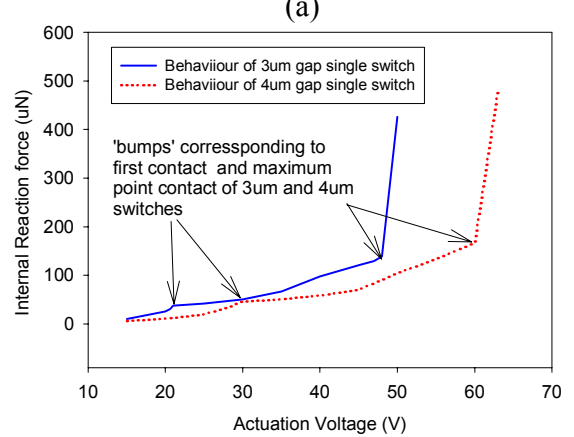

(b)

Fig. 3. a) Displacements (b) Reaction forces against the actuation voltage. Graphs show relationships with different gap separation

\section{B. Case 2}

For electromechanically modelling of the partially linked quadruplet switch configuration of Fig. 1(c), we have used a basic model which is the head-to head joined double switch as shown in Fig. 4(a). The 3D simulation for this bridge structure also shows as the actuation voltage increases, the beam bending up to a full contact, see Fig. 4(b), and further deforming resulting in the buckling as shown in Fig. 4(c). 


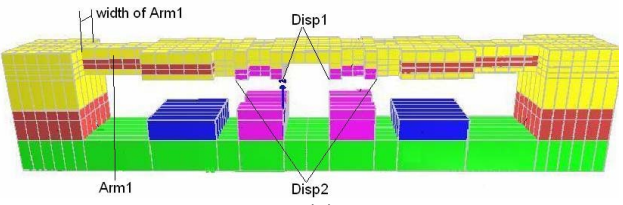

(a)

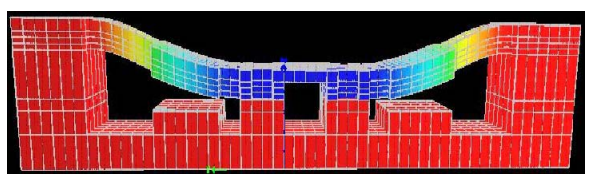

(b)

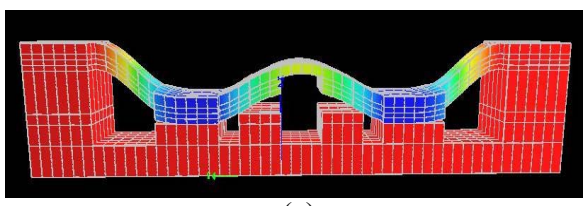

(c)

Fig. 4. \{a) Double switch model (unactuated). (b) Full contact state. (c) Buckling state.

Fig. 5(a) plots the displacements of two points on the beam, i.e. disp 1 and disp 2 as indicated in Fig. 4(a), against the actuation voltage for a case when the initial beam separation is $3 \mu \mathrm{m}$. The stage 1 is before the beam making the first contact with the RF transmission line. The stage 2 is for the beam making a full RF contact. The stage 3 maintains the full contact, and this is the desired range of operational voltage. The stage 4 is when the switch buckling occurs and there is no more full contact.

From the reaction force versus voltage graph of Fig. 5(b), we can observe that there is a region of linearity from the point of first touch through the maximum operational voltage. In order to have good RF contact, and contact force, it is our interest to improve this range of operational voltage. It has to be noted that the reaction force stated is defined as the force experienced externally by the switch arm.

It is envisaged that the contact force experienced by the switch contacts cannot be easily obtained due to the many variables involved. We have divided the bending process into 4 different stages as described above. The reaction force at the end of stage 1 , denoted by $F_{1}$ is taken to be the spring force of the beam for the first ohmic contact. The reaction force $F_{2}$ obtained at the end of stage 2 the force needed to bend the beam for a full ohmic contact. The third stage involves the point of full contact up to the maximum operational voltage, or the point of buckling when the beam experience the reaction force $F_{3}$. At this full-contact stage, the contact force will be propotional to

$$
\Delta F=F_{3}-F_{2}
$$

The larger the $\Delta F$, the larger the contact force is with a full ohmic contact.

The fourth stage is the buckling stage. This is the failure stage and full contact no longer exists. From Fig 5(b), we can observe that the buckling and switch collapse occurs in this stage and the reaction force increases sharply and becomes non-linear.

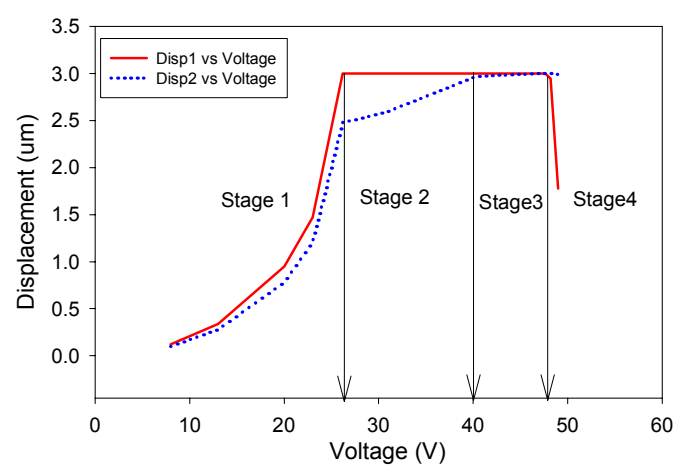

(a)

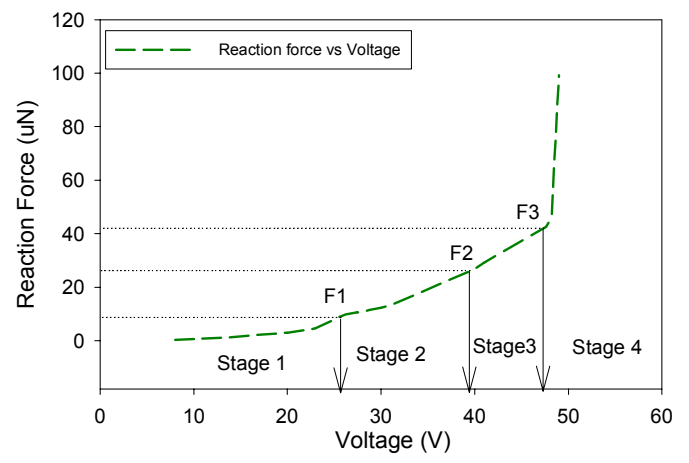

(b)

Fig. 5. (a) Showing displacements of the two RF contacts versus actuation voltage. (b) Showing external reaction forces experienced on the edge of switch versus actuation voltage

In order to have a larger $\Delta F$ for a better ohmic contact, we have investigated a critical dimension of the bridge beam, namely, the width of the arm 1 as indicated in Fig. 4(a). The width is varied and the results shown in Table 1(a) for the actuation voltages at the ends of first three stages. While the reaction forces at the ends of those stages are listed in Table 1(b).

\begin{tabular}{|c|c|c|c|}
\hline $\begin{array}{r}\text { Width of } \\
\text { Arm1 }(\mu \mathrm{m})\end{array}$ & $\begin{array}{c}\text { First Touch } \\
(\mathrm{V})\end{array}$ & $\begin{array}{c}\text { Full Touch } \\
(\mathrm{V})\end{array}$ & $\begin{array}{c}\text { Max Point } \\
(\mathrm{V})\end{array}$ \\
\hline 40 & 24 & 38 & 46 \\
\hline 50 & 26 & 40 & 48.2 \\
\hline 60 & 28 & 41.5 & 50 \\
\hline
\end{tabular}

Table 1. (a) Showing the effect of width on the actuation voltage

\begin{tabular}{|c|c|c|c|c|}
\hline $\begin{array}{l}\text { Width of } \\
\text { Arm1 }(\mu \mathrm{m})\end{array}$ & $\mathrm{F} 1(\mu \mathrm{N})$ & $\mathrm{F} 2(\mu \mathrm{N})$ & $\mathrm{F} 3(\mu \mathrm{N})$ & $\Delta \mathrm{F}(\mu \mathrm{N})$ \\
\hline 40 & 8.56 & 24.8 & 41 & 16.2 \\
\hline 50 & 9.5 & 26.8 & 45.7 & 18.9 \\
\hline 60 & 11 & 29 & 52 & 23 \\
\hline
\end{tabular}

Table 1. (b) Showing the effect of width on the reaction force.

From Table 1(a), we observe that an increment of $10 \mu \mathrm{m}$ width on the switch arm (Arm1) has the effect of increasing the first touch, full touch and maximum point voltage by about $2 \mathrm{~V}$. Hence from the width-voltage relationship seems to be linear. Table 1(b) shows that, for a $10 \mu \mathrm{m}$ increment in width of Arm1, and thus a linear width-reaction force relationship is not observable. Table 1(b) also shows that $\Delta \mathrm{F}$ increases with increasing width 
of Arm1. Hence, it points to the conclusion that a larger width will improve the contact force experienced by the contacts. However, the trade-off in this case would be higher actuation voltage required by the switch.

\section{EXPERIMENT}

The following arrangement was established to verify the actuation performance of the Quadruple switch. The die was epoxy bonded using Ablefilm ${ }^{\mathrm{TM}}$ 5025E onto a brass housing. A Teflon piston applied pressure from a weight assembly to the die during the curing cycle. A recess in the piston face ensured protection of the switches during this process. The processes for the assembly is illustrated in Fig. 6.

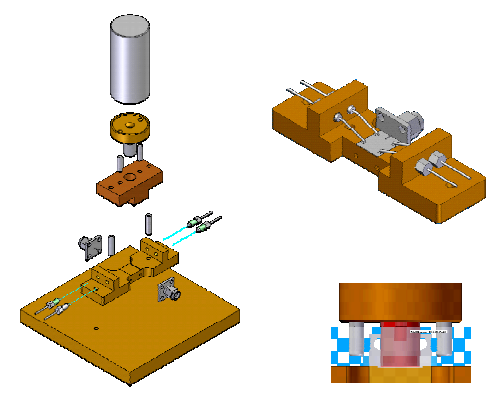

Fig. 6. Showing die mount assembly and connections.

The die is $10 \mathrm{~mm}$ by $9 \mathrm{~mm}$ including the $\mathrm{DC}$ bias circuits as shown Fig. 7. The DC contacts were made using fine wire springs that were soldered to axial feed throughs. The springs were then rotated into place and epoxy bonding to the DC pads on the die. The RF connections were made using Huber Suhner SMA connectors with $.2 \mathrm{~mm}$ centre pins. These were directly epoxy bonded to the microstrip feed lines. The DC test setup is shown in Fig. 8 below.

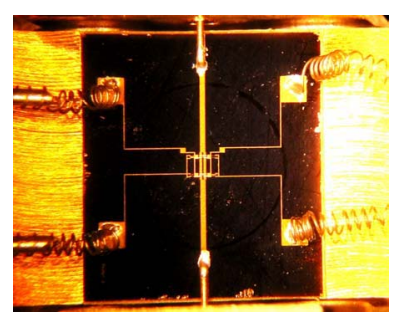

Fig. 7. Switch Die mounted on holder with DC and RF connections.

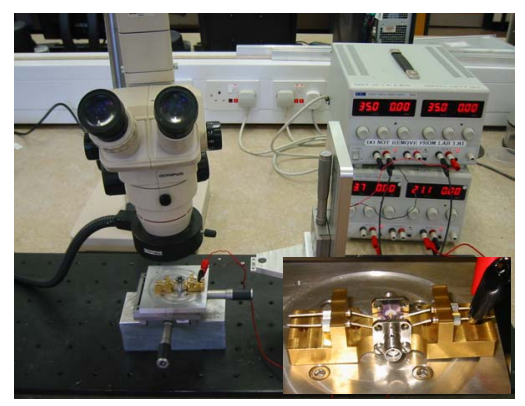

Fig. 8. DC test setup.
The switch was observed using an Olympus SZ40 microscope. The DC voltage was switched between 0 and positive 70 . Actuation occurred at $35.8 \mathrm{~V}$ for one of the four switches. A second switch actuates at $50 \mathrm{~V}$ and all four can be observed to actuate at $70 \mathrm{~V}$. The recorded actuation voltage for the first switch agrees with the theory presented. The increased voltages required for the other switches may be explained by increased gap spacing due to the stresses present after the beam release stage. The material stresses remain to be optimised in the fabrication process.

\section{CONCLUSION}

The electromechanical modelling of RF MEMS switch configurations for high power handling has been presented. Using a 3D electromechanical simulator allows one to examine the insight of electromechanical behaviours. It has revealed the switch beams can suffer from the buckling at a higher actuation voltage due to deformation. For a better ohmic contact, maintaining full contact with a larger contact force is essential. It has been shown that increasing the width of beam could achieve this with the trade-off for the increase in DC actuation voltage. A preliminary experiment has been carried out for DC testing a quadruplet RF MEMS switch. The tested DC actuation voltages were in the range of the predicted ones by simulation.

\section{ACKNOWLEDGEMENT}

The financial support from the EPSRC for the project (GR/S68910/01) on High power RF/microwave MEMS switches is acknowledged.

\section{REFERENCES}

[1] G. M. Rebeiz, RF MEMS-Theory, Design and Technology, John Wiley \& Sons.,Inc., 2003.

[2] P. Blondy, M. Farina, P. Farinelli, P. Mezzanotte, R. Sorrentino, "A New Single-Pole Double-Throw MEMS Switch Topology", Proceedings of the 34th European Microwave Conference, Amsterdam, NL, October 2004, pp. 1429-1431.

[3] N. Nishijima, J. Hung, G.M Rebeiz, "A low-voltage high contact force rf-mems switch "IEEE MTT-S Symposium Digest, Vol 2, June 6-11, 2004 pp. $577-580$

[4] X. Rottenberg, S. Brebels, B. Nauwelaers, R.P. Mertens, W. Raedt, and H. A. C. Tilmans, "Modelling of the RF self-actuation of electrostatic RF-MEMS devices", Micro Electro Mechanical Systems, 2004. $17^{\text {th }}$ IEEE International Conference on. (MEMS), 2004, pp.245-248.

[5] N. Nishijima, Juo-Jung Hung, and G. M. Rebeiz, "Parallelcontact metal-contact RF-MEMS switches for high power applications", Micro Electro Mechanical Systems, 2004. 17th IEEE International Conference on. (MEMS) , 2004 pp.781-784.

[6] B. D. Jensen, K. Jensen, J. L. Volakis, and K. Kurabayashi, "Fully integrated electrothermal multidomain modeling of RF MEMS switches", IEEE Microwave and Wireless Components Letters, Volume: 13 , No. 9, Sept. 2003, pp. 364-366.

[7] J.-S. Hong, S. G. Tan, et.al , "Development of high power rf mems switches", International Conference on Microwave and Millimeter Wave Technology, Beijing, August 2004, pp. 7-10. 